\title{
Cardiac diffusion tensor imaging based on compressed sensing using joint sparsity and low-rank approximation
}

\author{
Jianping Huang ${ }^{\mathrm{a}, \mathrm{b}}$, Lihui Wang ${ }^{\mathrm{c}}$, Chunyu Chu ${ }^{\mathrm{a}}$, Yanli Zhang ${ }^{\mathrm{a}}$, Wanyu Liu ${ }^{\mathrm{a}, \mathrm{b}, *}$ and \\ Yuemin $\mathrm{Zhu}^{\mathrm{a}, \mathrm{b}}$ \\ ${ }^{a}$ Metislab, LIA CNRS, Harbin Institute of Technology, Harbin, Heilongjiang, China \\ ${ }^{\mathrm{b}}$ CREATIS, INSA Lyon, INSA Lyon, University of Lyon, Villeurbanne, France \\ ${ }^{\mathrm{c}}$ College of Computer Science and Technology, Guizhou University, Guiyang, Guizhou, China
}

\begin{abstract}
Diffusion tensor magnetic resonance (DTMR) imaging and diffusion tensor imaging (DTI) have been widely used to probe noninvasively biological tissue structures. However, DTI suffers from long acquisition times, which limit its practical and clinical applications. This paper proposes a new Compressed Sensing (CS) reconstruction method that employs joint sparsity and rank deficiency to reconstruct cardiac DTMR images from undersampled k-space data. Diffusion-weighted images acquired in different diffusion directions were firstly stacked as columns to form the matrix. The matrix was row sparse in the transform domain and had a low rank. These two properties were then incorporated into the CS reconstruction framework. The underlying constrained optimization problem was finally solved by the first-order fast method. Experiments were carried out on both simulation and real human cardiac DTMR images. The results demonstrated that the proposed approach had lower reconstruction errors for DTI indices, including fractional anisotropy (FA) and mean diffusivities (MD), compared to the existing CS-DTMR image reconstruction techniques.
\end{abstract}

Keywords: Diffusion tensor imaging, compressed sensing, low rank, sparsity constraint

\section{Introduction}

Diffusion tensor magnetic resonance (DTMR) imaging or diffusion tensor imaging (DTI) are MRI techniques that allow quantitative characterization of the geometry and organization of tissue microstructures. It is a powerful tool and is currently the only means to noninvasively investigate fiber architectures of the human heart [1]. However, DTI suffers from a long acquisition time and a low signal-to-noise ratio (SNR), which greatly limits its practical and clinical applications. Many attempts have been made to enhance the efficiency and effectiveness of SNR of diffusion signals [2-5].

Recently, Compressed Sensing (CS) has emerged as a new framework for reconstructing high quality signals from fewer samples than required by the traditional Shannon-Nyquist sampling theorem $[6,7]$. Based on the principles of CS, this paper proposes a constrained reconstruction technique based on a regularization framework to jointly reconstruct sparse sets of cardiac DTI data [8].

\footnotetext{
${ }^{*}$ Corresponding author: Wanyu Liu, Metislab, LIA CNRS, Harbin Institute of Technology, 92 West Dazhi Street, Nan Gang District, Harbin 150001, Heilongjiang, China. Tel.: +86 0451 86403287; E-mail: Liu_wanyu@ @it.edu.cn.
}

0928-7329/16/\$35.00 @ 2016 - IOS Press and the authors. All rights reserved This article is published online with Open Access and distributed under the terms of the Creative Commons Attribution NonCommercial License. 
Considering that the diffusion weighted (DW) images obtained at different gradient directions are often correlated, a distributed compressed sensing-based method offers an alternative to reconstructing images one-by-one [9]. This technique exploits joint sparsity among DW images to obtain better reconstruction quality. In addition, the DW images are stacked as matrix column vectors; the resulting matrix is rank-deficient. CS-DTMR image reconstruction from undersampled $\mathrm{k}$-space is then formulated as a low-rank matrix approximation problem [10]. On the other hand, a model-based CS method for DTI was proposed in [11], which uses the signal intensity model to directly estimate diffusion tensor fields from undersampled $\mathrm{k}$-space data.

This paper proposes a new CS reconstruction method that employs joint sparsity and rank deficiency prior to cardiac DTMRI reconstruction. DW images acquired at different gradient directions are firstly stacked as columns to form the matrix; the matrix is row sparse in the transform domain and also has a low rank. These two properties are then incorporated into the CS reconstruction framework. The underlying optimization problem is finally solved by the first-order fast method.

This paper is organized as follows: Section 2 presents the proposed reconstruction method; Section 3 analyzes the method's performance using simulation and real human cardiac datasets; Section 4 discusses the conclusion.

\section{Theory and algorithm}

Assuming that $x_{l}$ is a DW image with size $m \times n$, and $F_{l}^{u}$ is a partial Fourier transform for the $l$ th direction, the undersampled k-space data $b_{l}$ of the DW image for each direction in k-space can be formulated as:

$$
b_{l}=F_{l}^{u} x_{l}+\varepsilon
$$

where $\varepsilon$ is the noise level and $l=1,2, \ldots, L$.

Since the DW images are correlated across diffusion directions, each image $x_{l}$ shares the same sparse support in the pixel or transform domain with other images. When the different images are stacked as column vectors of matrix $X$, the resulting matrix is row sparse. Exploiting the joint sparsity property along diffusion directions can achieve better reconstruction accuracy [9].

$$
X_{m n \times L}=\left[x_{1}, x_{2}, \ldots, x_{l}, \ldots, x_{L}\right]=\left[\begin{array}{cccc}
x_{1,1} & x_{1,2} & \ldots & x_{1, L} \\
x_{2,1} & x_{2,2} & \ldots & x_{2, L} \\
\vdots & \vdots & \ldots & \vdots \\
x_{m n, 1} & x_{m n, 2} & \ldots & x_{m n, L}
\end{array}\right]
$$

This paper proposes a new reconstruction method for DW images by combining row sparse and lowrank prior properties of the data matrix $X$. Consequently, the reconstruction can be described with the following optimization problem:

$$
\widehat{X}=\underset{x}{\arg \min }\left\{\frac{1}{2}\left\|F^{u} X-b\right\|_{2}^{2}+\alpha \cdot\|X\|_{*}+\beta \cdot\|\Phi X\|_{2,1}\right\} .
$$

here, $\Phi$ is a sparsity transform, such as wavelet transform; $\alpha>0$ and $\beta>0$ are two regularization parameters, which trade off the relative importance of the joint sparsity and the low-rank constraint. 
Equation (3) can be solved by the Fast Composite Splitting Algorithm (FCSA) proposed in [12]. Specifically, the sub-problem can be solved by a proximal operation:

$$
\operatorname{prox}_{\rho}(\phi)(x)=\arg \min \left\{\phi(u)+\frac{1}{2 \rho}\|u-x\|_{2}^{2}\right\}
$$

The outline for the proposed method for problem (3) is as follows:

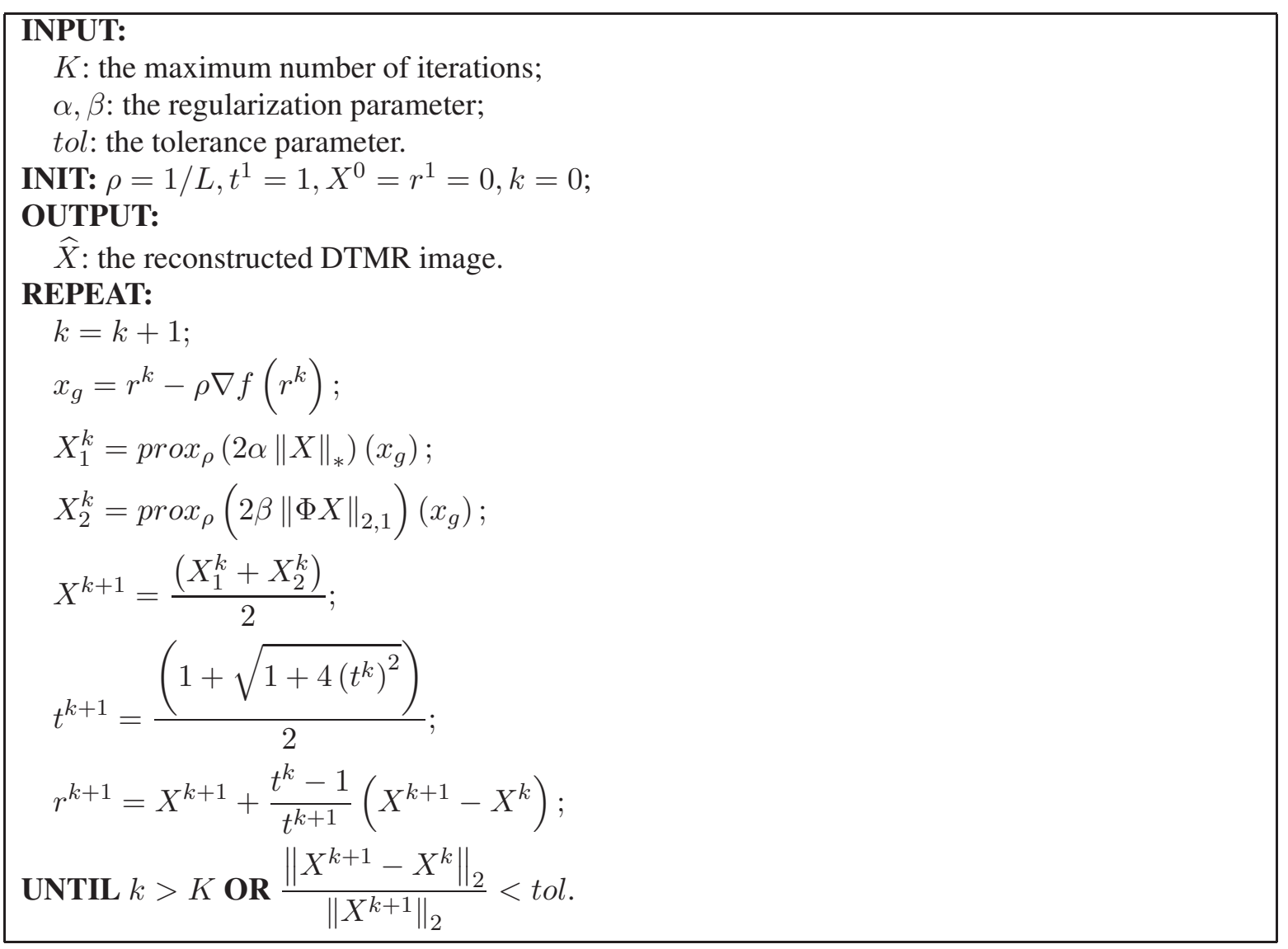

\section{Experimental results}

Simulated DW images were generated as proposed in [13], which were obtained with diffusion gradient directions applied in 42 directions. The real human heart datasets were acquired with a Siemens Avanto 1.5 T Scanner and using a diffusion spin-echo EPI sequence with 12 diffusion gradient directions; it was repeated 6 times to enhance SNR. The size of the diffusion-weighted images was $128 \times 128$ (image spatial resolution was $2.0 \times 2.0 \times 2.0 \mathrm{~mm}^{3}$ ). The simulated and real DW images were obtained with diffusion sensitivity $b=1000 \mathrm{~s} / \mathrm{mm}^{2}$ and a $128 \times 128$ image size. The k-space was sampled using the variable density undersampling pattern [14].

To evaluate the performance of the proposed method, a comparison was performed with several stateof-the-art reconstruction methods, including the basic CS method [12], low rank [15], and joint sparsity method [9]. The root mean square errors (RMSE) of fractional anisotropy (FA) and mean diffusivity 


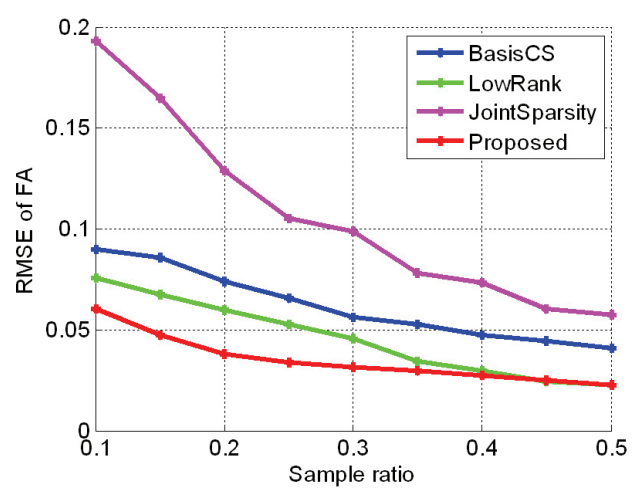

(A)

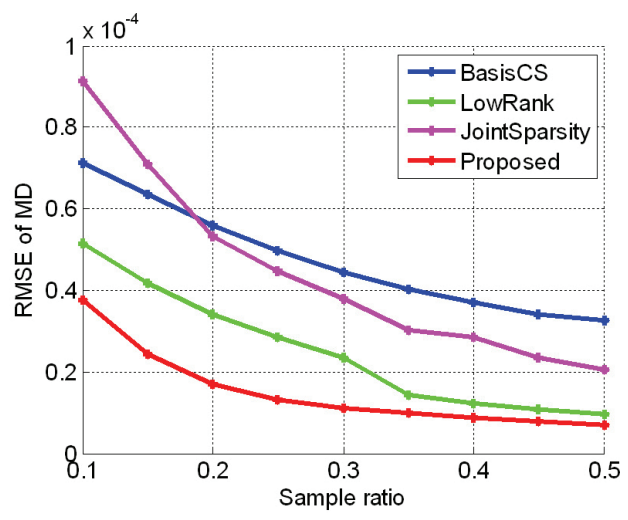

(B)

Fig. 1. Performance comparisons on simulated data with different sampling rates. (A) RMSE of FA; (B) RMSE of MD.

(MD) were calculated for each method. The Fractional anisotropy (FA) and mean diffusivity (MD) are respectively defined by:

$$
\begin{aligned}
\mathrm{MD} & =\frac{\lambda_{1}+\lambda_{2}+\lambda_{3}}{3} \\
\mathrm{FA} & =\sqrt{\frac{3 \cdot\left[\left(\lambda_{1}-\mathrm{MD}\right)^{2}+\left(\lambda_{2}-\mathrm{MD}\right)^{2}+\left(\lambda_{3}-\mathrm{MD}\right)^{2}\right]}{2 \cdot\left(\lambda_{1}^{2}+\lambda_{2}^{2}+\lambda_{3}^{2}\right)}}
\end{aligned}
$$

where $\lambda_{1}, \lambda_{2}$, and $\lambda_{3}$ refer to the eigenvalues of the diffusion tensor.

The root mean square errors (RMSE) is calculated as follows:

$$
\operatorname{RMSE}=\sqrt{\frac{\left\|\operatorname{vec}\left(x_{r e c}\right)-\operatorname{vec}\left(x_{r e f}\right)\right\|_{2}^{2}}{N}}
$$

where, $x_{r e c}$ and $x_{r e f}$ denote respectively the reconstructed signal and reference signal, and $N$ is the total number of signal.

The observation measurement $b$ is corrupted by complex Gaussian white noise $\varepsilon$ with standard deviation $\sigma_{n}$. The latter is derived from the associated input SNR (ISNR), which is defined as ISNR = $20 \log _{10}\left(\sigma_{x} / \sigma_{n}\right)$, where $\sigma_{x}$ denotes the standard deviation of the DW images. The Daubechies wavelets with four decomposition levels are used; the ISNR is set as $20 \mathrm{~dB}$; the parameter $\alpha$ and $\beta$ are set to 0.1 and 0.0035 , respectively.

\subsection{Effects of sampling rates}

Figures 1 and 2 compare the reconstruction performance of the four methods with sampling rates of $10 \% \sim 50 \%$ for simulated and real human cardiac data.

From the figures, it can be seen that the proposed method achieved better reconstructions with lower RMSE of FA and MD compared with other methods.

Table 1 gives the RMSE of FA and MD using different methods on simulated and real data with a $20 \%$ sample ratio. 
Table 1

RMSE of FA and MD with sample ratio $20 \%$ (The unit of MD is $\times 10^{-3} \mathrm{~mm}^{2} / \mathrm{s}$ )

\begin{tabular}{lcccc}
\hline & FA (simulated data) & MD (simulated data) & FA (real data) & MD (real data) \\
\hline Basis CS & 0.074 & 0.056 & 0.057 & 0.061 \\
Low rank & 0.060 & 0.034 & 0.031 & 0.036 \\
Joint sparsity & 0.129 & 0.053 & 0.032 & 0.034 \\
Proposed & 0.038 & 0.017 & 0.025 & 0.026 \\
\hline
\end{tabular}

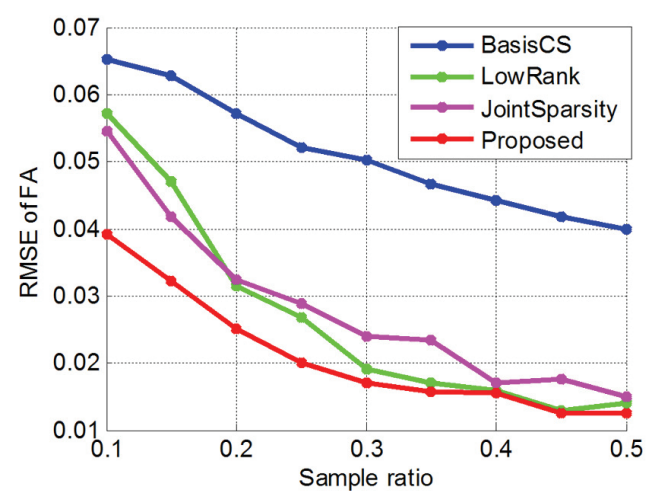

(A)

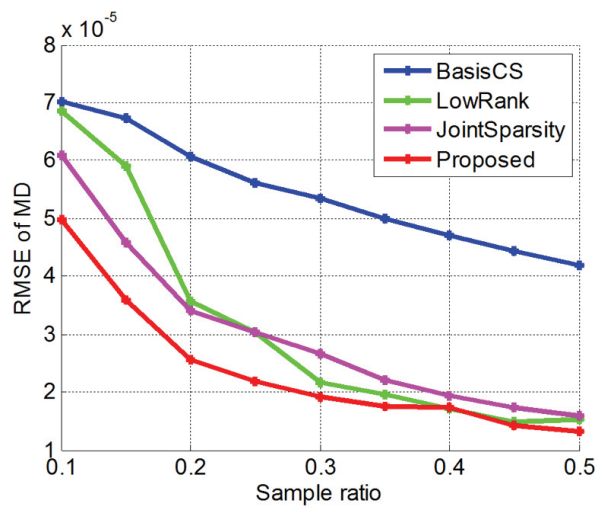

(B)

Fig. 2. Performance comparisons on real data with different sampling rates. (A) RMSE of FA; (B) RMSE of MD.

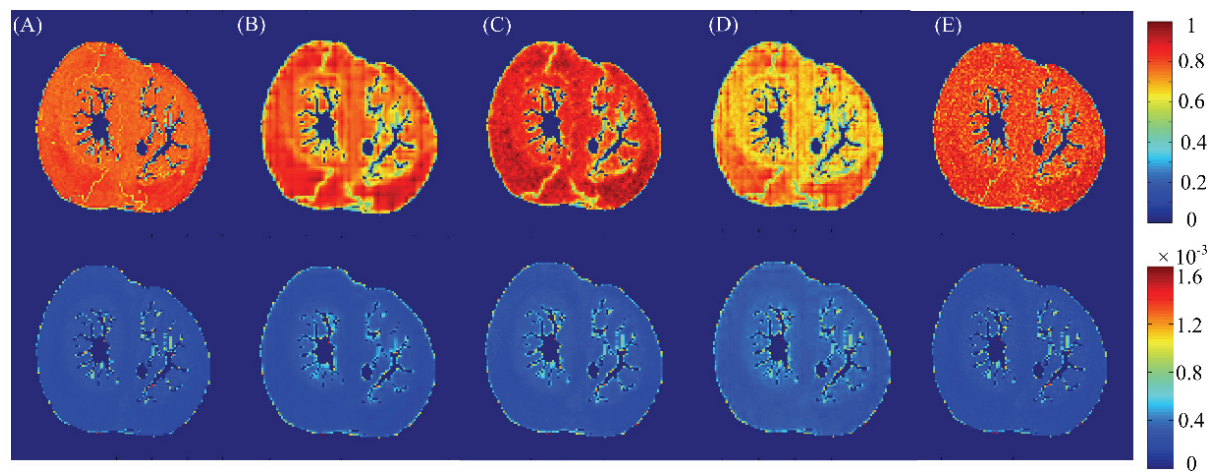

Fig. 3. Maps of FA (first row) and MD (second row) of the simulated human heart data with $25 \%$ sampling rates. (A) Reconstruction from the complete k-space data. Reconstructions from undersampled k-space using (B) basis CS, (C) low rank, (D) joint sparsity, and (E) the proposed methods.

\subsection{Maps of FA and MD}

Figures 3 and 4 map FA and MD on simulated and real heart data with a 25\% sampling rate, which corresponds to an acceleration factor of 4 . In these calculations, the involved diffusion tensors corresponding to the complete k-space were taken as references. As shown in Figs 3 and 4, the FA and MD maps with the proposed method are visually better than those obtained with other methods.

\subsection{Effects of regularization parameters}

The regularization parameters $\alpha$ and $\beta$ were empirically determined. Different values were tested 


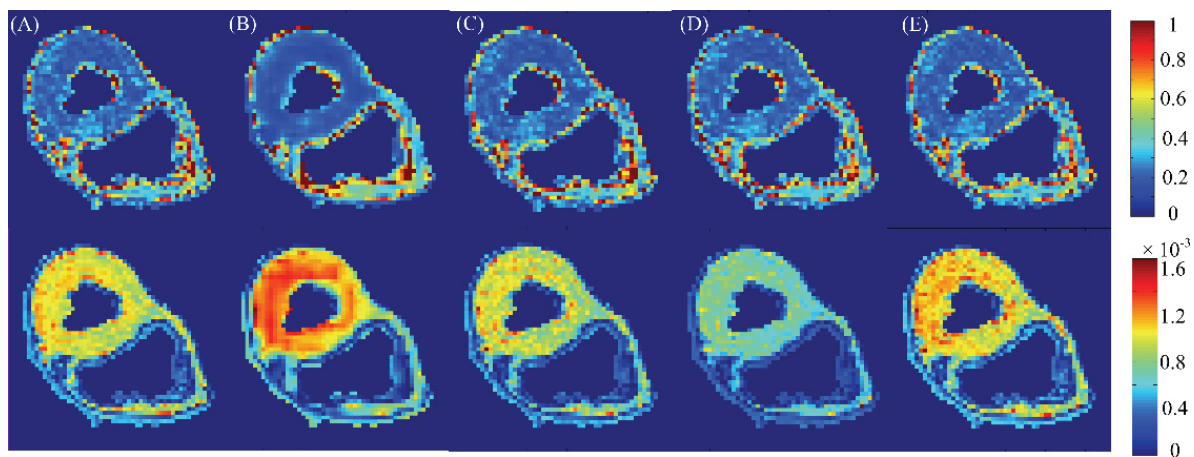

Fig. 4. Maps of FA (first row) and MD (second row) of the real human heart data with $25 \%$ sampling rates. (A) Reconstruction from the complete k-space data. Reconstructions from undersampled k-space using (B) basis CS, (C) low rank, (D) joint sparsity, and (E) the proposed methods.

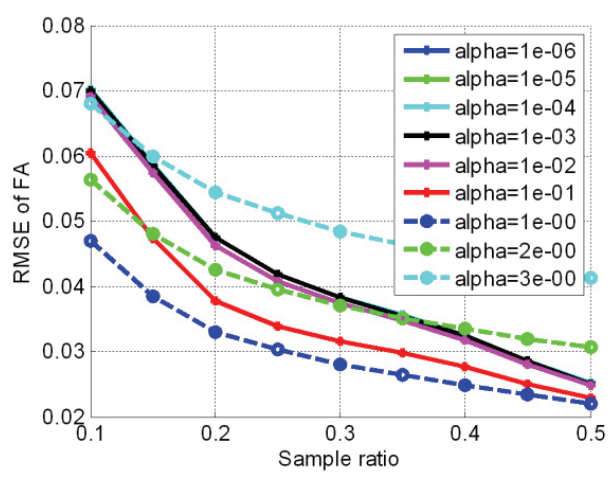

(A)

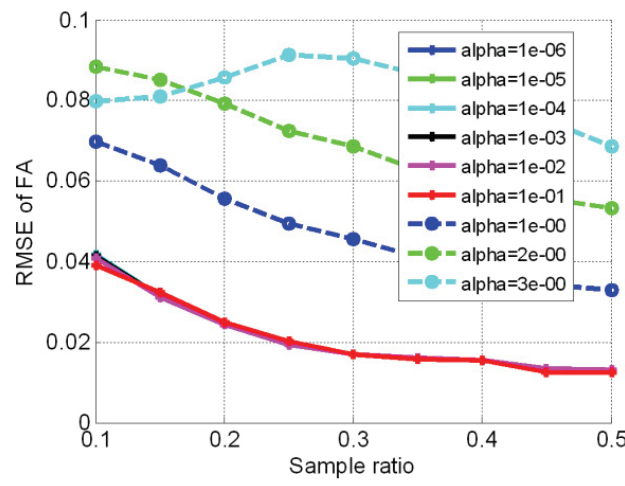

(C)

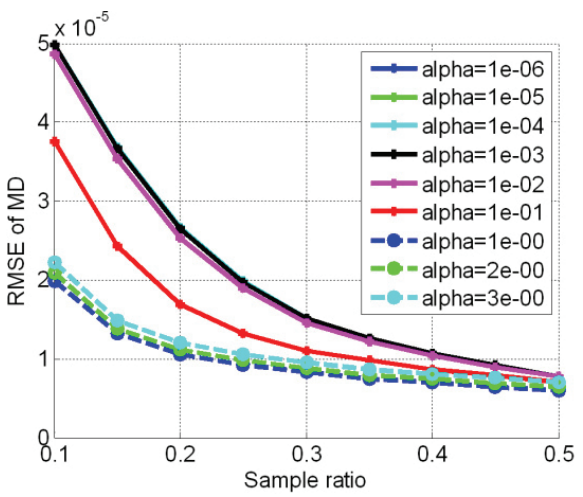

(B)

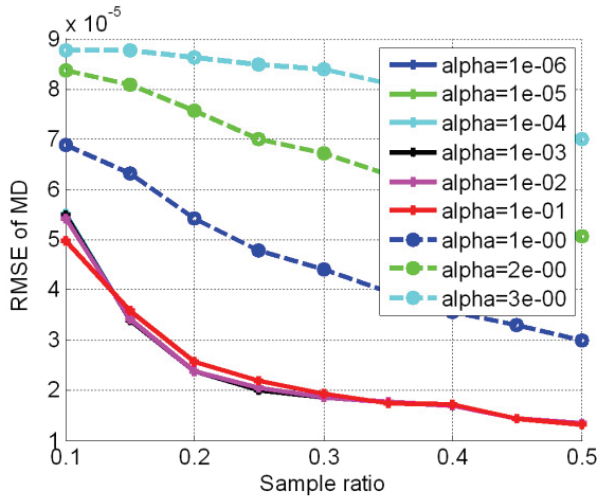

(D)

Fig. 5. Effects of regularization parameters. (A) RMSE of FA on simulated data; (B) RMSE of MD on simulated data; (C) RMSE of FA on real data; (D) RMSE of MD on real data.

for $\alpha$ (fixing $\beta=0.0035$ ). As illustrated in Figs 5A and B, the reconstruction RMSE of FA and MD on simulated data changed slightly when $\alpha$ was smaller than 1e-02. As shown in Figs $5 \mathrm{C}$ and $\mathrm{D}$, the reconstruction RMSE of FA and MD on real human heart data change slightly when $\alpha$ was smaller than 1e-01. Based on these results, $\alpha$ and $\beta$ were set to 0.1 and 0.0035 , respectively. 


\section{Conclusion}

This paper proposed an efficient method for reconstructing DTMR images from undersampled $\mathrm{k}$-space data using compressed sensing. Based on the combined use of joint sparsity and low rank regularization, the proposed method exploits simultaneously intra-image spatial redundancy and inter-image correlation across diffusion directions. The results on both simulated and real human cardiac DW images showed that the proposed method provides more accurate reconstructions and more accurate DTI indices compared with the state-of-the-art CS DTMR image reconstruction techniques.

\section{Acknowledgments}

This work was supported in part by the National Natural Science Foundation of China (No. 61271092), the applied technology research and development program of Heilongjiang Province (No. GC13A311), the Inter-Government S\&T Cooperation Project between P.R. China and Romania (No. 40-20), the Program PHC-Cai Yuanpei 2012, the French ANR under ANR-13-MONU-0009-01, the Funds for Talents of Guizhou University (No. 2013(33)) and the Nature Science Foundation of Guizhou province (No. 20152044).

\section{References}

[1] D. Le Bihan, J. F. Mangin, C. Poupon, C. A. Clark, S. Pappata, N. Molko, and H. Chabriat, Diffusion tensor imaging: Concepts and applications, Journal of Magnetic Resonance Imaging, vol. 13, pp. 534-546, Apr 2001.

[2] Y. Jiang and E. W. Hsu, Accelerating MR diffusion tensor imaging via filtered reduced-encoding projectionreconstruction, Magnetic Resonance in Medicine, vol. 53, pp. 93-102, Jan 2005.

[3] J. P. Haldar, V. J. Wedeen, M. Nezamzadeh, G. Dai, M. W. Weiner, N. Schuff, and Z.-P. Liang, Improved diffusion imaging through SNR-enhancing joint reconstruction, Magnetic Resonance in Medicine, vol. 69, pp. 277-289, Jan 2013.

[4] S. J. Holdsworth, S. Skare, R. D. Newbould, and R. Bammer, Robust GRAPPA-accelerated diffusion-weighted readoutsegmented (RS)-EPI, Magnetic Resonance in Medicine, vol. 62, pp. 1629-1640, Dec 2009.

[5] R. Bammer, M. Auer, S. L. Keeling, M. Augustin, L. A. Stables, R. W. Prokesch, R. Stollberger, M. E. Moseley, and F. Fazekas, Diffusion tensor imaging using single-shot SENSE-EPI, Magnetic Resonance in Medicine, vol. 48, pp. 128-136, Jul 2002.

[6] E. J. Candes, J. Romberg, and T. Tao, Robust uncertainty principles: Exact signal reconstruction from highly incomplete frequency information, IEEE Transactions on Information Theory, vol. 52, pp. 489-509, Feb 2006.

[7] D. L. Donoho, Compressed sensing, IEEE Transactions on Information Theory, vol. 52, pp. 1289-1306, Apr 2006.

[8] G. Adluru, E. Hsu, and E. V. R. Di Bella, Constrained reconstruction of sparse cardiac MR DTI data, in Functional Imaging and Modeling of the Heart, Proceedings, vol. 4466, F. B. Sachse and G. Seemann, Eds., ed, 2007, pp. 91-99.

[9] Y. Wu, Y.-J. Zhu, Q.-Y. Tang, C. Zou, W. Liu, R.-B. Dai, X. Liu, E. X. Wu, L. Ying, and D. Liang, Accelerated MR diffusion tensor imaging using distributed compressed sensing, Magnetic Resonance in Medicine, vol. 71, pp. 763-772, Feb 2014.

[10] L. Li, X. Hu, and H. Gao, Compressive Diffusion MRI-Part 1 Why Low-Rank, in Proceedings of the 21th Annual Meeting of ISMRM, Salt Lake, 2013.

[11] C. L. Welsh, E. V. R. DiBella, G. Adluru, and E. W. Hsu, Model-based reconstruction of undersampled diffusion tensor k-space data, Magnetic Resonance in Medicine, vol. 70, pp. 429-440, Aug 2013.

[12] J. Huang, S. Zhang, and D. Metaxas, Efficient MR image reconstruction for compressed MR imaging, Medical Image Analysis, vol. 15, pp. 670-679, Oct 2011.

[13] L. Wang, Y. Zhu, H. Li, W. Liu, and I. E. Magnin, Multiscale modeling and simulation of the cardiac fiber architecture for DMRI, IEEE Transactions on Biomedical Engineering, vol. 59, pp. 16-19, Jan 2012.

[14] M. Lustig, D. Donoho, and J. M. Pauly, Sparse MRI: The application of compressed sensing for rapid MR imaging, Magnetic Resonance in Medicine, vol. 58, pp. 1182-1195, Dec 2007.

[15] S. G. Lingala, Y. Hu, E. DiBella, and M. Jacob, Accelerated dynamic MRI exploiting sparsity and low-rank structure: k-t SLR, IEEE Transactions on Medical Imaging, vol. 30, pp. 1042-1054, May 2011. 